\title{
A Dual-Frequency Miniaturized Frequency Selective Surface Structure Suitable for Antenna Stealth
}

\author{
Wei Zhang, Maojun Li, Mingnan Le $\mathbb{D}^{\text {, }}$ Bin Li, and Jiaqi Wei \\ School of Information Science and Technology, Northwest University, Xi'an, CO 710127, China \\ Correspondence should be addressed to Mingnan Le; lemingnan@nwu.edu.cn
}

Received 1 October 2021; Revised 16 November 2021; Accepted 25 November 2021; Published 27 December 2021

Academic Editor: Guangwei Yang

Copyright (C) 2021 Wei Zhang et al. This is an open access article distributed under the Creative Commons Attribution License, which permits unrestricted use, distribution, and reproduction in any medium, provided the original work is properly cited.

\begin{abstract}
A tiny dual-band frequency selective surface structure is proposed in this paper. With dual-band rejection characteristics at the corresponding frequency points of the S-band and C-band, suitable for antenna stealth. To achieve miniaturization, the unitcell architecture resembles the shape of a "S." First of all, the author describes the parameters of the surface element, and then, the transmission characteristics of the surface element are analyzed by the equivalent circuit method. By maintaining a constant response to TE and TM polarization patterns and oblique incident angles, the suggested device ensures angular independence. The measured findings from the constructed FSS are used to validate the computed results. Finally, a new unit structure is provided for the application of FSS in antenna stealth.
\end{abstract}

\section{Introduction}

The traditional structure-type wave-absorbing material Salisbury screen is composed of an impedance layer $\lambda / 4$ away from the metal backplane, a space layer, and a connection base plate. The Salisbury screen has a simple structure, but its narrow absorption bandwidth and the need for a larger thickness at low frequencies restrict the application of this structure in practical engineering [1]. The Jaumann absorber is improved on the basis of the traditional Salisbury screen. It is composed of multiple dielectric layers and impedance layers. Although the absorption bandwidth has been greatly improved, the thickness of the absorber is increased, and the structure is also increased. The design difficulty and complexity limit its practical application. In order to improve the comprehensive performance of absorbing materials to meet actual needs, absorbing materials based on frequency-selective surfaces have become the research focus. FSS has a variety of patterns, which can be optimized by loading lumped elements or materials with specific impedance, and the resonance frequency can be modified by controlling the size of the FSS and the loaded resistance. Compared with the Salisbury screen, it can only pass through in terms of adjusting the thickness of the media layer, and the design of the absorber based on the frequency selection surface is more diversified.

An endless periodic array of metal patches or apertures is known as a frequency selective surface. The features of the FSS mainly depend on the shape and dimension of the FSS unit. Common FSS units are square, ring, or crossed dipoles, which are widely used in radome, beam steering, radar cross-section reduction, and wireless security [2-5]. Antenna stealth technology is advancing at a breakneck pace. The frequency selection of the antenna on the drone surface technology has also been widely used. Unmanned aerial vehicles are widely used in air operations due to they do not require combatants to directly face various dangers. At present, there are strong military and political use requirements; in order to meet the needs of these fields, we need to continuously improve its design and performance.

In 2008, $\mathrm{Xu}$ and others realized the frequency selection surface of resonance at $2.5 \mathrm{GHz}$ and $3.5 \mathrm{GHz}$ by loading lumped capacitors [6]. Sivasamy and Kanagasabai [7] and Chen et al. [8] led the team and successfully developed a dual-band FSS structure with low-frequency ratio, which was used in practical applications. In order to accommodate more FSS unit structures in a limited space, a miniaturized design of FSS structures will be required. Sheng et al. [9] and Li et al. 
[10] have reduced the size of FSS units by designing curved unit structures to achieve the purpose of miniaturization, however, for antenna stealth, miniaturization alone is not enough. We also need to ensure its angular stability. Another frequent way to improve angular performance is to use multilayer technology. The coupling of two meander line resonators separated by an ultrathin substrate results in a compact FSS element [11]. Up to 80 degrees, the FSS can create a consistent angular response. In recent years, the teams of Ghosh and Srivastava [12], Hong Tao et al. [13], and Tong et al. [14] have designed a bent multicurrent path structure to obtain a dualband frequency selective surface with a compressed cell structure and good angular stability. These studies provide a useful reference for the design of dual-frequency and miniaturized FSS structures.

This paper is mainly for antenna stealth. A new type of miniaturized dual-frequency structure is proposed, which can realize the band rejection characteristics of S-band 3.8 $\mathrm{GHz}$ and C-band $5.0 \mathrm{GHz}$ through two dual-frequency points, and the characteristics of FSS are verified through physical tests. The results show that the designed structure not only has two closely adjacent resonance points that can be adjusted independently but also has the advantages of miniaturization and incident angle stability up to $60^{\circ}$.

\section{FSS Design}

2.1. Unit-Cell Design. AS we all know, the angular stability of the FSS is affected by both the unit-cell spacing and the element's structure. Generally speaking, the basic element with symmetric structure has better angular stability and smaller cell size than the basic element with asymmetric structure, but the unit structure adopted in this article is asymmetric. The proposed structure is the modification of cross ring, and the improved cross-shaped ring unit is composed of two mutually perpendicular " $S$ "-shaped rings. The "S"shaped ring is composed of two circular rings with different radii and the same width. The center of the ring unit does not coincide with the center of the upper surface of the dielectric substrate. Figure 1 depicts the unit-cell geometry, the material of the substrate is FR-4 with relative permittivity $\varepsilon_{\gamma}=4.4$, loss tangent $\tan \delta=0.02$, and thickness $h=$ $2.56 \mathrm{~mm}$. Figure 2 shows the FSS surface shape, and fullwave simulations are implemented using the software HFSS Microwave Studio. Table 1 displays the optimal settings, where $D$ is the length of the unit period, $g$ is the distance between the units, $W$ is the width of the ring, and $\alpha$ is the central angle.

2.2. Analysis Frequency Selection Surface. The analysis method of FSS is an important content of FSS research. At present, the analysis methods of FSS mainly include pattern matching method, spectral domain method, numerical method, and equivalent circuit method. This article mainly uses equivalent circuit method to analyze FSS structure, ignoring the thickness of the metal layer, each metal layer and its underlying dielectric layer can be regarded as an FSS layer, and the bottom metal layer alone is regarded as an FSS layer, then the structure is a two-layer FSS structure.

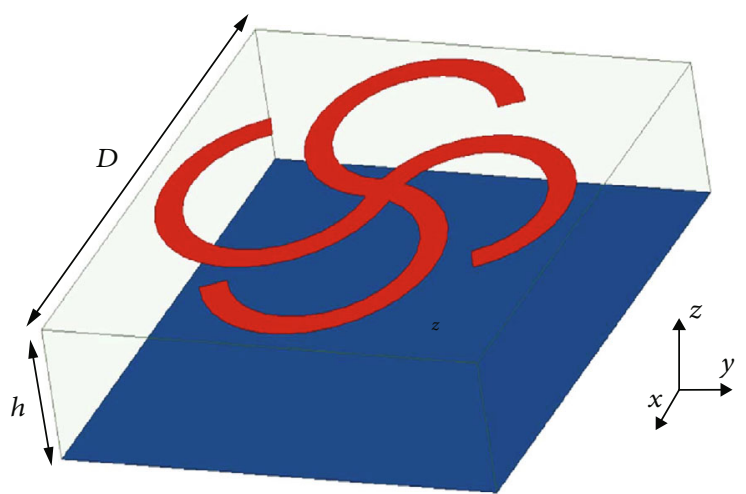

FIgURE 1: Proposed unit-cell geometry.

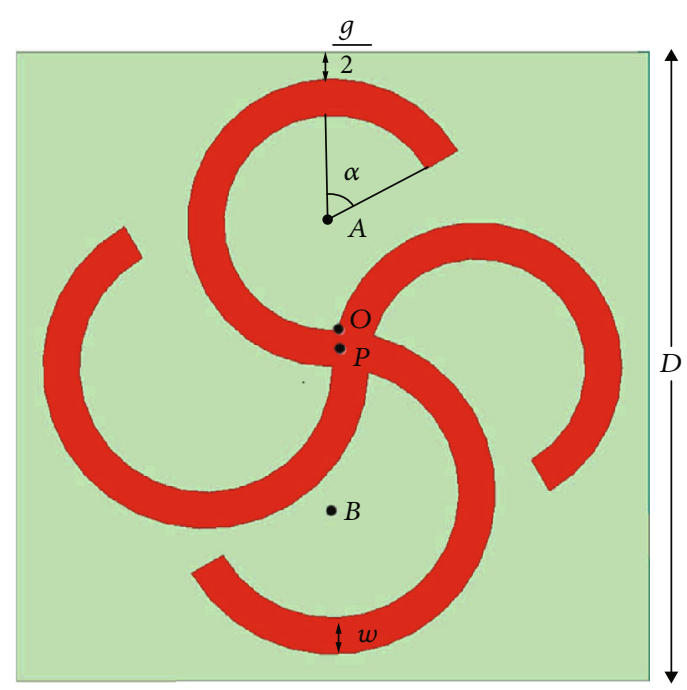

FIgURE 2: Related dimensions of upper surface structure.

According to transmission line theory, the equivalent circuit model for this two-layer FSS structure is shown in Figure 3.

In this circuit, $Z_{0}$ and $\beta_{0}$ separately denote the peculiarity impedance and dissemination constant in free space; $Z_{d}$ and $\beta_{d}$ denotes the peculiarity impedance and dissemination constant in the medium; $Z_{m 1}$ represents the equivalent impedance of the fss metal layer, $Z_{m 2}$ denotes the equivalent impedance of the bottom metal patch. So we can get

$$
\begin{aligned}
\beta_{0} & =\frac{w}{c}, \\
\beta_{d} & =\beta_{0} \sqrt{\varepsilon_{r}(1-j \tan \delta)}, \\
Z_{0}^{T E} & =\frac{\eta_{0}}{\cos \theta}, \\
Z_{0}^{T M} & =\eta_{0} \cos \theta, \\
Z_{d} & =\frac{\eta_{0}}{\sqrt{\varepsilon_{r}(1-j \tan \delta)}},
\end{aligned}
$$

where $w$ is the angular velocity, $c$ is the speed of light in vacuum, $\tan \delta=0.02$ represents the loss tangent of the 
TABLE 1: Geometric parameters of the FSS.

\begin{tabular}{lccccccc}
\hline Parameter & $D$ & $h$ & $g$ & $w$ & $\alpha$ & OA & OB \\
\hline Value & $11.2 \mathrm{~mm}$ & $2.56 \mathrm{~mm}$ & $0.96 \mathrm{~mm}$ & $0.64 \mathrm{~mm}$ & $60^{\circ}$ & $2.24 \mathrm{~mm}$ & $2.56 \mathrm{~mm}$ \\
\hline
\end{tabular}

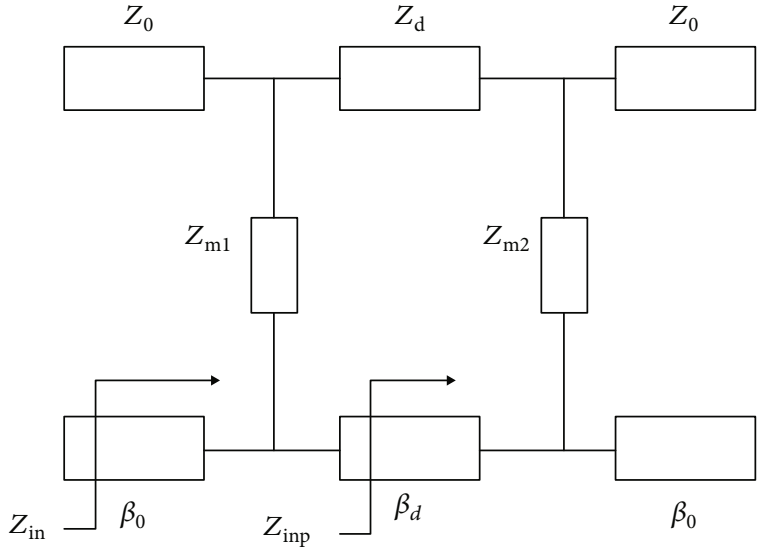

Figure 3: Circuit model of transmission line.

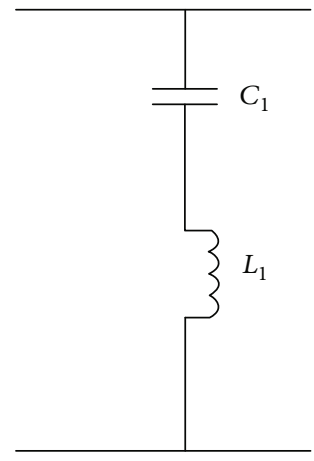

Figure 4: Equivalent circuit facsimile of the metal layer.

dielectric layer, and the wave impedance in open space is represented by $\eta_{0}$.

The equivalent input impedance of the entire two-layer FSS structure can be expressed as

$$
\begin{aligned}
Z_{\text {in }} & =Z_{0} \frac{Z_{L}+j Z_{0} \tan (\beta h)}{Z_{0}+j Z_{L} \tan (\beta h)}, \\
\Gamma & =\frac{Z_{L}-Z_{0}}{Z_{L}+Z_{0}}, \\
\tau^{2}+\Gamma^{2} & =1 .
\end{aligned}
$$

In the formula, $h$ represents the thickness of the medium, and $Z_{L}$ represents the effective load impedance. The transmission coefficient $\tau$ can be calculated.

The metal layer shown in Figure 4 can be equivalent to the form of a series resonant circuit L1-C1 in the transmis-

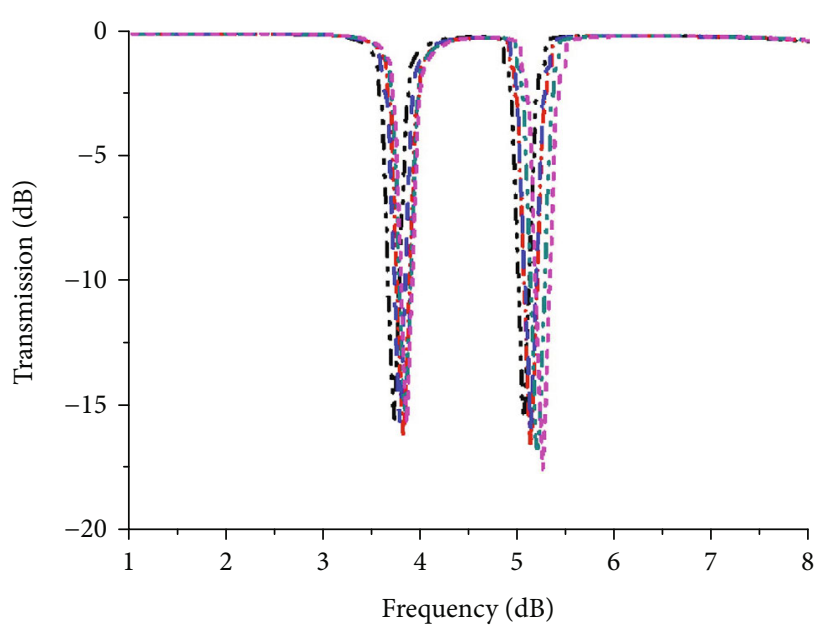

TE mode

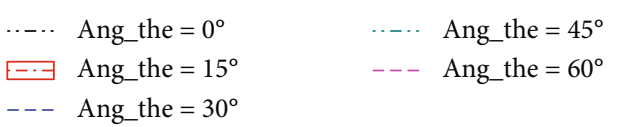

FIgURE 5: TE wave absorption at different angles of incidence.

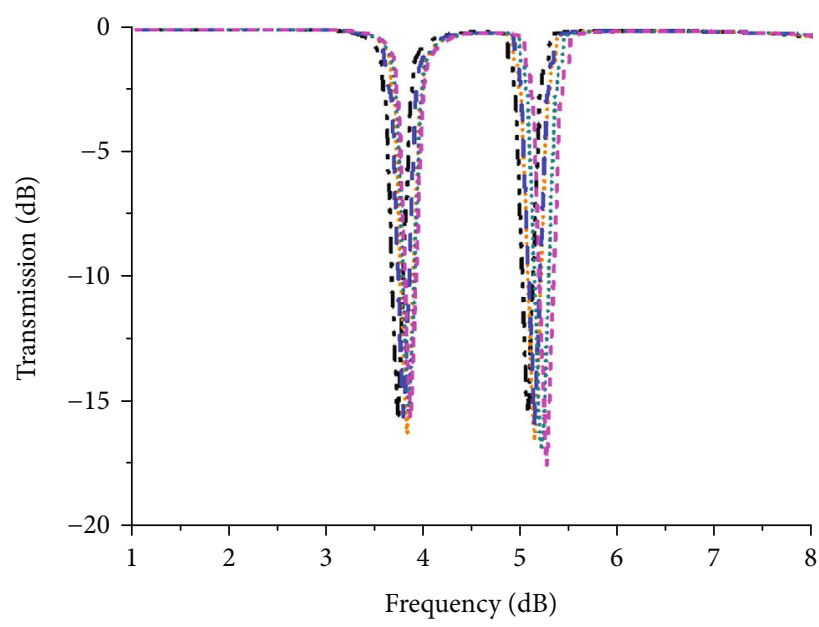

TM mode

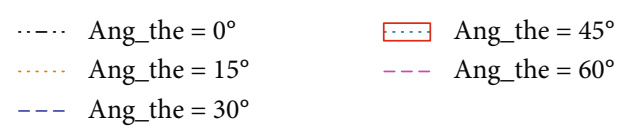

FIgURE 6: TM wave absorption at different angles of incidence.

sion line model, and the normalized inductance and capacitance of the component are [15]

$$
X_{L}=\frac{p}{D} F(D, 2 w, \lambda)
$$




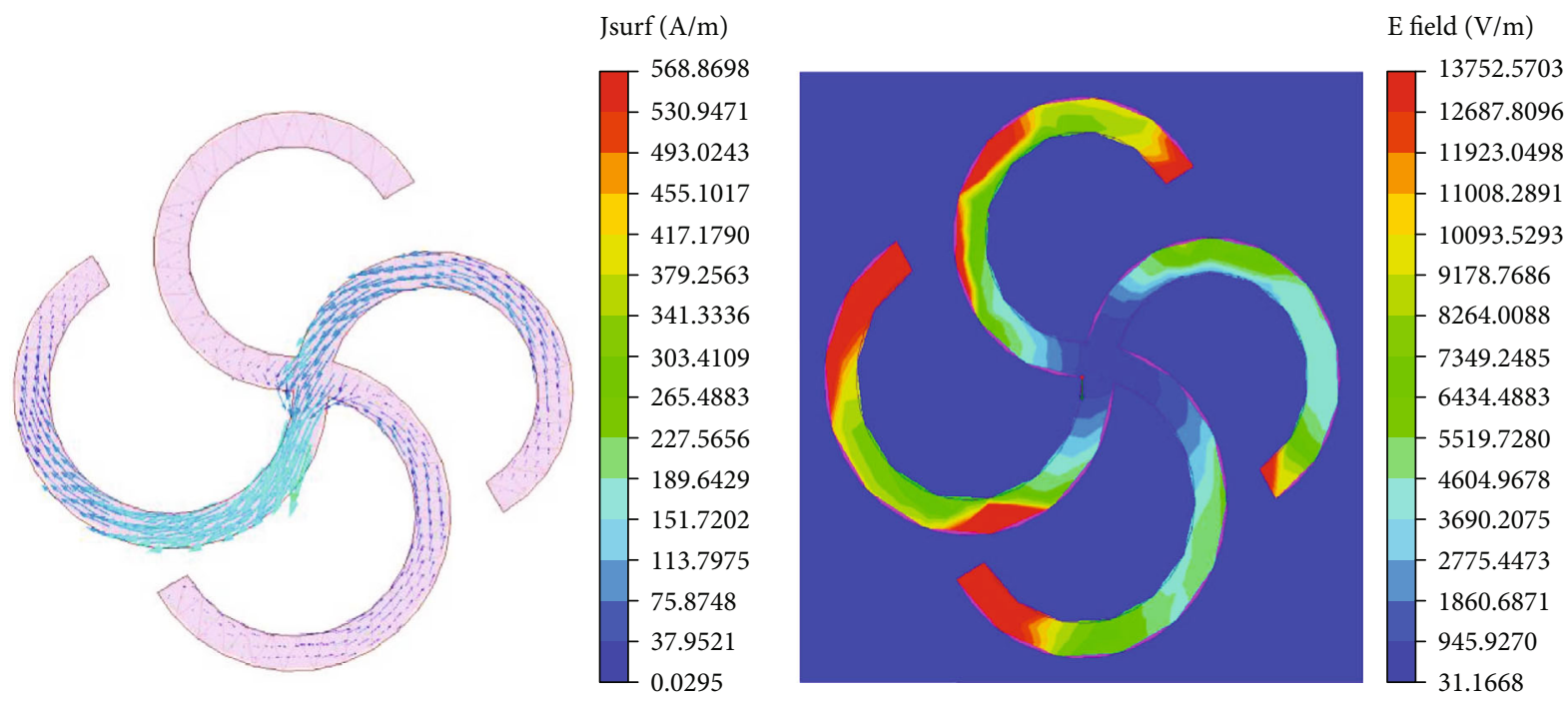

Figure 7: $3.8 \mathrm{GHz}$ field strength distribution in the metal layer.
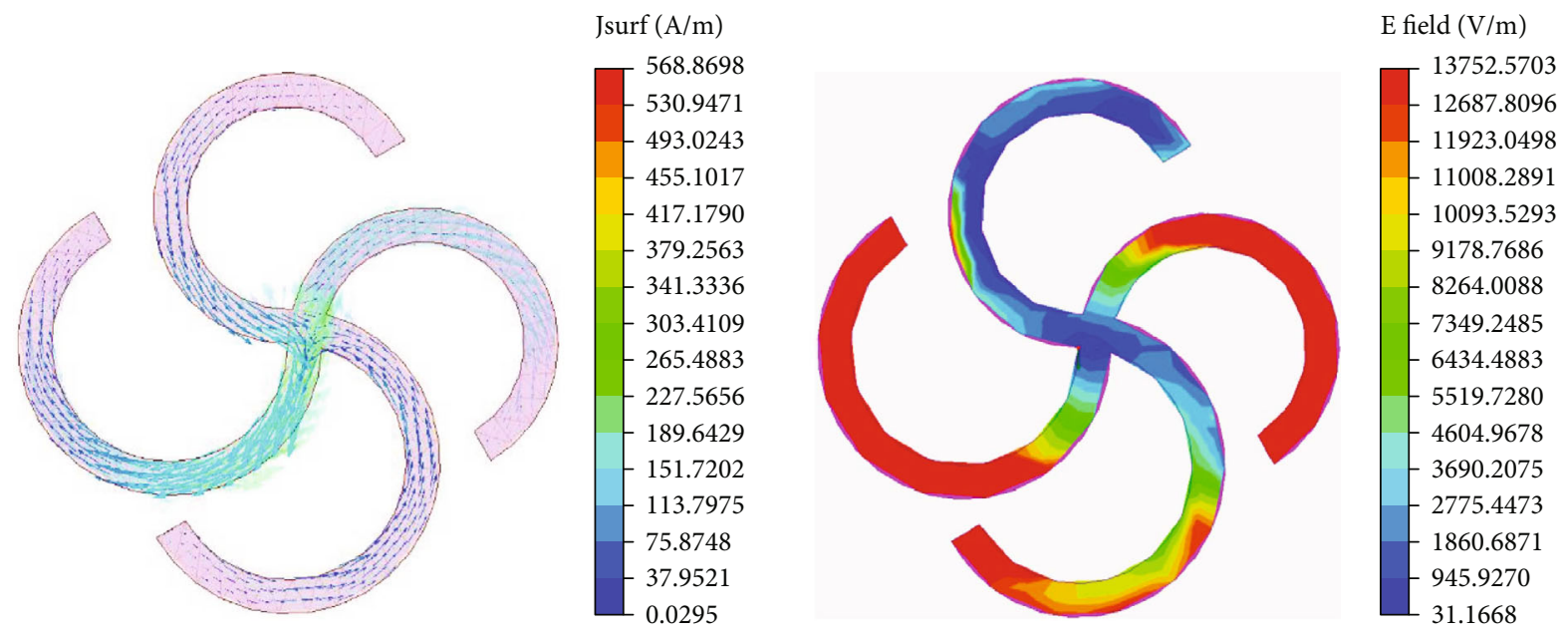

FIGURE 8: $5.0 \mathrm{GHz}$ field strength distribution in the metal layer.

$$
\begin{gathered}
X_{C}=\frac{4 p}{D} \varepsilon_{e f f} F(D, g, \lambda), \\
F(D, w, \lambda)=\frac{D}{\lambda}\left\{\operatorname{In} \csc \left\{\frac{\pi w}{2 D}\right\}+G(D, w, \lambda)\right\} \\
G(D, w, \lambda)=\frac{1}{2} \frac{\left(1-\beta^{2}\right)^{2}\left\{\left(1-\beta^{2} / 4\right)\left(C_{+}+C_{-}\right)+4 \beta^{2} C_{+} C_{-}\right\}}{\left(1-\beta^{2} / 4\right)+\beta^{2}\left(1+\left(\beta^{2} / 2\right)-\beta^{4} / 8\right)\left(C_{+}+C_{-}\right)+2 \beta^{6} C_{+} C_{-}} .
\end{gathered}
$$

The capacitive reactance is affected when the frequency selection unit is loaded with a medium. Assume that $\varepsilon_{e f f}$ is the medium's equivalent relative dielectric constant. When calculating the multilayer structure, $\varepsilon_{e f f}=1+\varepsilon_{r} / 2$ for the first and last layer, and $\varepsilon_{e f f}=\varepsilon_{r}$ for the middle layer. $p$ represents the side length of the $S$ ring, $g$ represents the cell gap, $w$ represents the width of the ring, $D$ represents the length of the smallest periodic element, and $\lambda$ is the wavelength of the incident electromagnetic wave.
Where

$$
c_{ \pm}=1 / \sqrt{1 \pm 2 D \sin \theta / \lambda-(D \cos \theta / \lambda)^{2}}-1
$$

$\beta=\sin \pi w / 2 D$. The normalized inductive reactance and normalized capacitive reactance of the FSS array can be determined through the calculation of the above formula, and the relevant electromagnetic parameters can be calculated by substituting in the theoretical calculation formula of the transmission line.

Figure 5 is the transmission curve of the FSS absorbing structure, where the minimum period $D=11.2 \mathrm{~mm}$, the loop width $w=0.64 \mathrm{~mm}$, and the cell gap $g=0.96 \mathrm{~mm}$. The material of the substrate is FR-4 with relative permittivity $\varepsilon_{\gamma=4.4}$, loss tangent $\tan \delta=0.02$, and thickness $h=2.56 \mathrm{~mm}$. It can be understood from Figure 5 that the transmission coefficient calculated by the equivalent circuit model is basically consistent with the HFSS simulation result, thus verifying the validity of the equivalent circuit model.

The practical implementation of FSS filters and shields needs FSS operation that is angular independent. The first thing we need to understand is that when a beam of light 


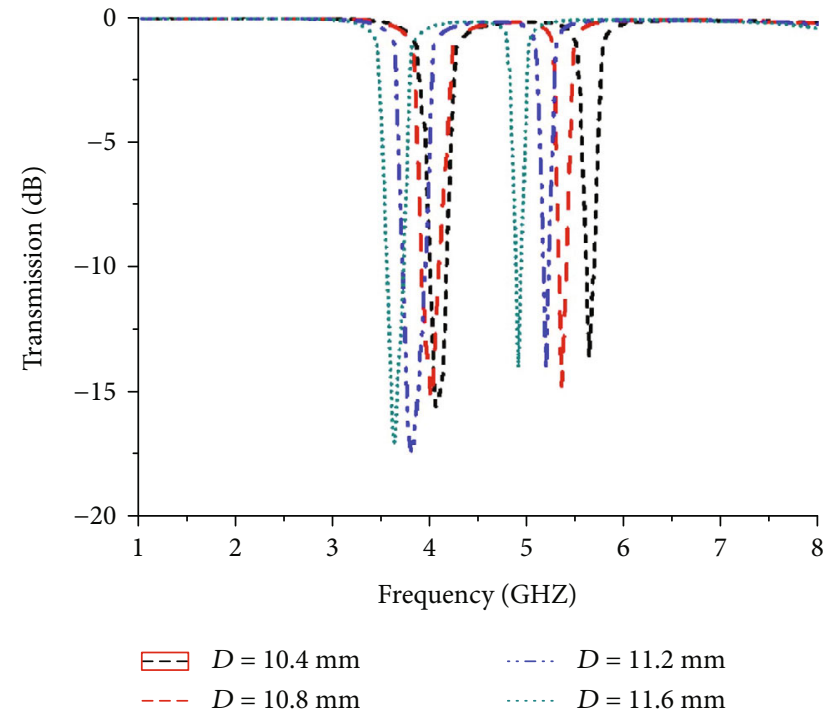

Figure 9: Absorption curve for different square ring size.

is incident on a metal surface, the plane where the incident light and the normal to the metal surface are located is the incident surface. The electric vector of the incident light wave can be decomposed into two polarized light components orthogonal to each other. TE waves are polarized light with a perpendicular electric vector to the incidence plane, whereas TM waves are polarized light with a parallel electric vector to the incident plane. The cells in a unit are illuminated with varying incident angles of $15^{\circ}, 30^{\circ}, 45^{\circ}$, and $60^{\circ}$ to demonstrate the design's angular stability, and the TMmode transmission peculiarity is presented in Figure 5.

The design gives a stable response for oblique impact angles, as seen in Figure 5. Similarly, the stability of the TE-mode transmission characteristics is presented in Figure 6. Through the analysis of the figure, we can get that whether in the TM mode or the TE mode, the frequency selection surface has two resonant frequency points $f_{1}=3.8$ $\mathrm{GHz}, f_{2}=5.0 \mathrm{GHz}$, and at 0 The frequency shift within the incident angle range of $0^{\circ} \sim 60^{\circ}$ does not exceed $0.5 \mathrm{GHz}$.

By observing the transmission curve, we can see that the absorber has two resonant frequencies, $3.8 \mathrm{GHz}$ and $5 \mathrm{GHz}$. When the electromagnetic wave enters the interior of the absorber, the field strength diagrams of the two frequency points are shown in Figure 7. As shown in Figure 8, after electromagnetic waves enter the absorbing body, strong electromagnetic resonance is generated, the energy is converted into heat energy and other forms of energy loss, and this is the absorbing principle of this design.

With the change of the size D of the FSS square ring, the transmission curve of the structure is shown in Figure 9. Obviously as the size of the square ring becomes larger and larger, the longer the current path, the absorption frequency band gradually shifts to low frequency. Therefore, by adjusting the square ring, the size is easy to adjust the absorption band of the structure, and the FSS resonance frequency point has a certain offset as the height $h$ grows, as seen in Figure 10 . We set $h=2.56 \mathrm{~mm}$ to ensure resonance at the desired frequency point. Similarly, analyzing the influence

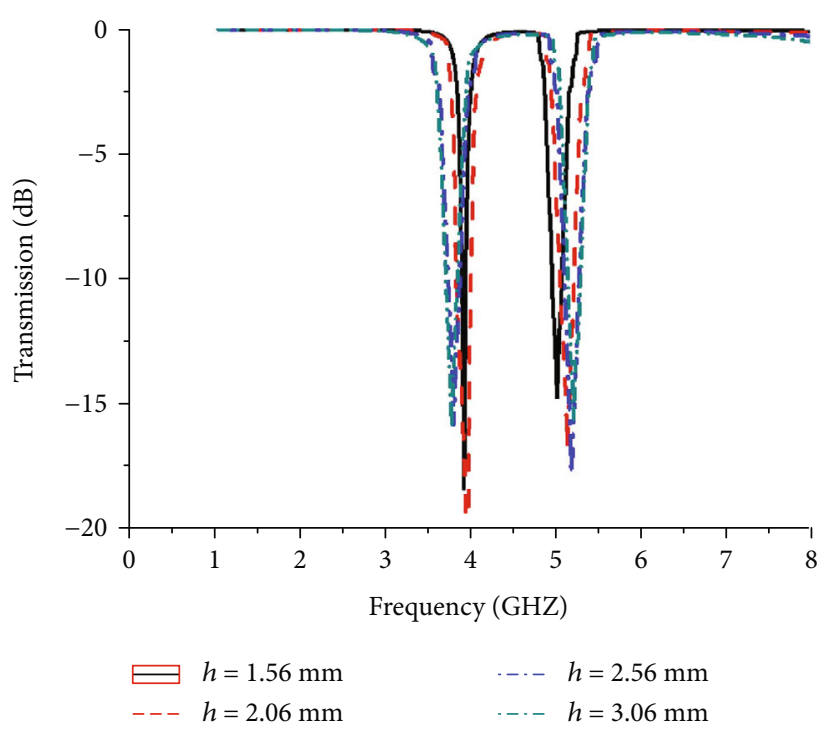

Figure 10: Absorption curves of different unit heights.

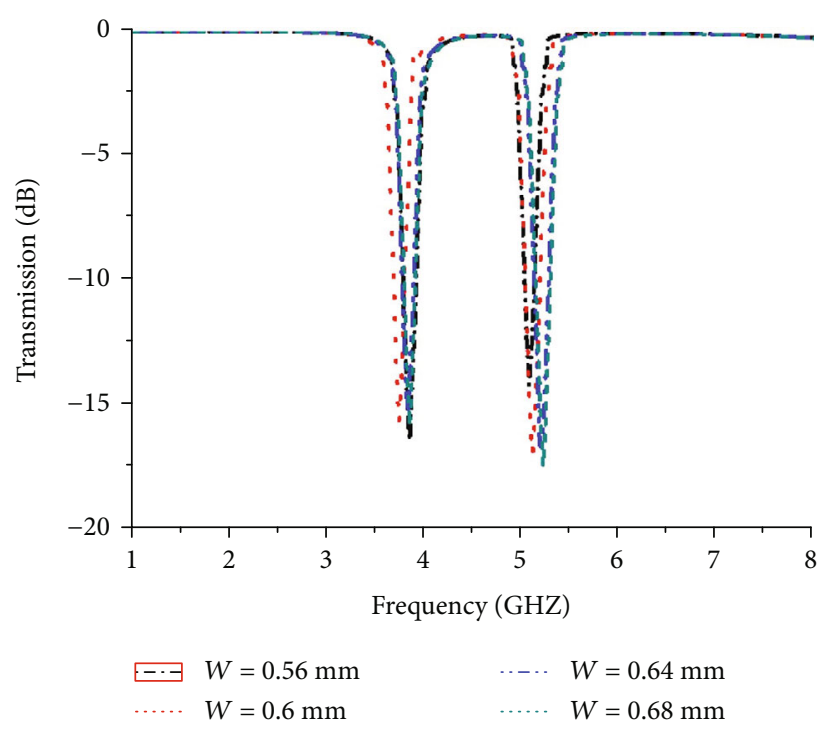

FIgURE 11: Absorption curve for different loop width.

of the loop width $w$ and the unit interval $g$ on the transmission curve, it can be seen from Figures 11 and 12 that the changes of these two parameters have little influence on the transmission.

2.3. Measurement Setup. We measured the free-space transmission of a prototype FSS to confirm the projected FSS performance. As illustrated in Figure 13, a prototype with dimensions of $112 \mathrm{~mm}$ is built and gauged in an anechoic enclosures.

Figure 14 depicts the measuring set-up. Use standard horn antennas WR229 and WR187 to connect the vector network subband to test the absorber. The tests were conducted in a semianechoic chamber. The range between the transmitting and receiving antennas and the FSS was 


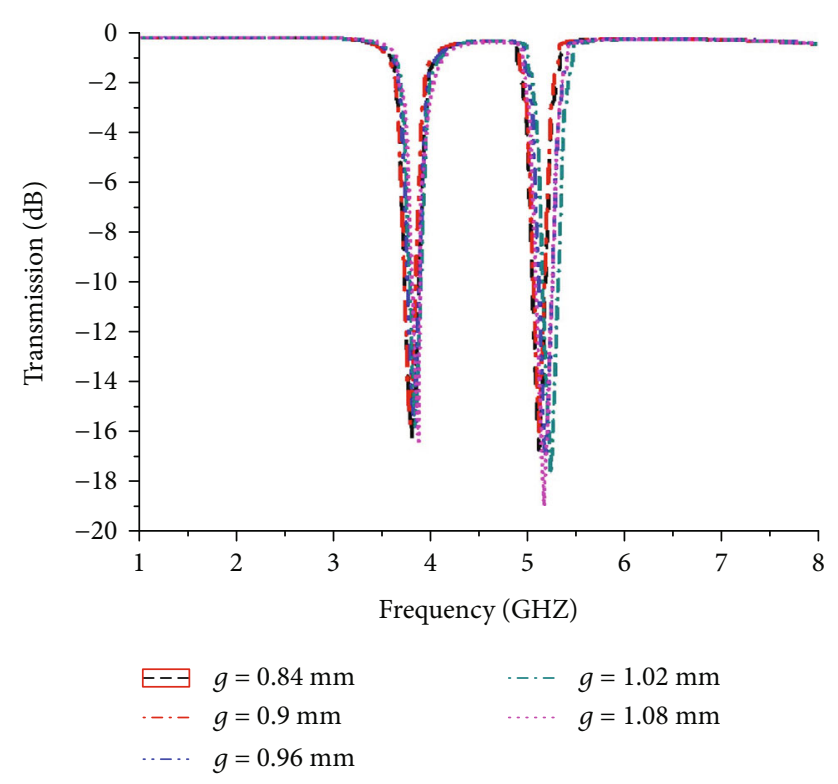

FIGURE 12: Absorption curve for different unit interval.

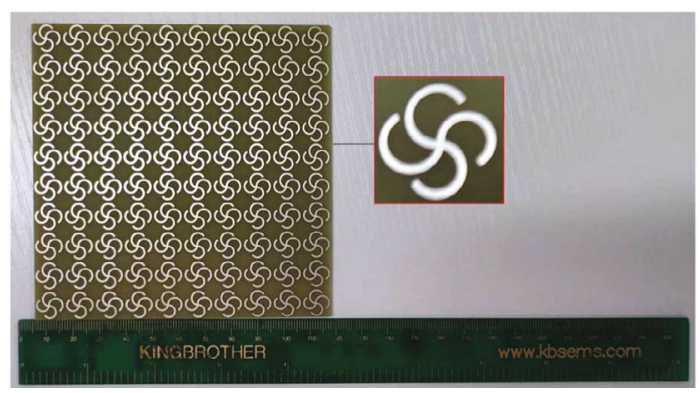

FIgURE 13: Fabricated prototype.

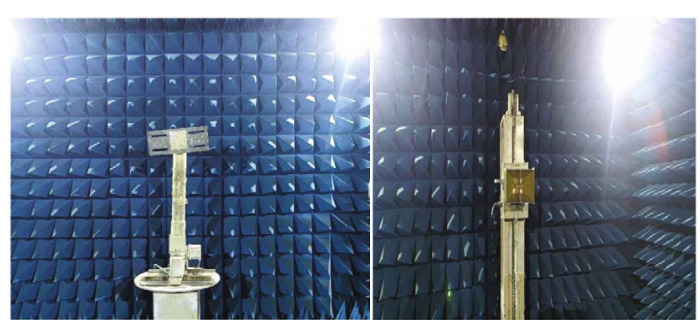

Figure 14: Test environment.

restricted to one meter. Absorbers were stored on the floor between the FSS and the transmitting antenna and on the side of the FSS facing the transmitting antenna. Figure 15 shows the measured and simulated values under normal incidence, indicating that the measured and simulated results are not completely coincident, and there is a certain difference, because the thickness of the air spacer is not permanent and is also related to the instability of the relative permittivity of the substrates, we assume that the variations between models and measurements are primarily due to this.

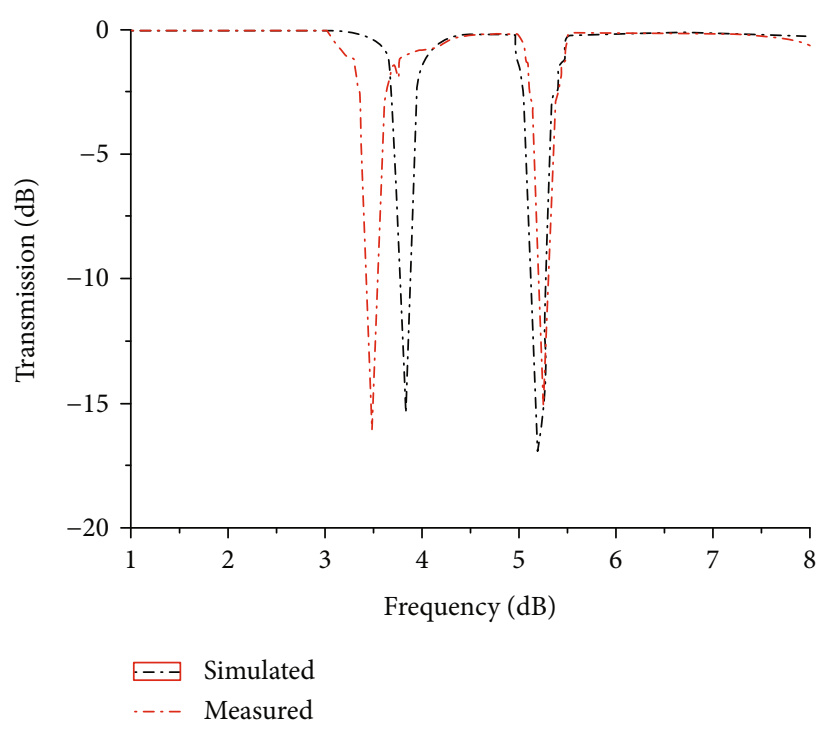

Figure 15: Measured and simulated transmissions.

\section{Conclusion}

A FSS element is proposed in this paper to attain miniaturized angular stability. Simulation and comparable current model analysis are used to investigate the compositional principle. Emulation and measurement are used to analyze FSS's performance. When the incidence angle varies from $0^{\circ}$ to $60^{\circ}$, the results demonstrate that steady angular performances are attained and the highest resonant frequency divergence is only $0.5 \mathrm{GHz}$. This FSS promises to be a viable choice for drone antenna stealth.

\section{Data Availability}

The data used to support the finding of this study are included in the article.

\section{Conflicts of Interest}

The authors declare that there are no conflicts of interest regarding the publication of this paper.

\section{Acknowledgments}

This work is supported by the National Natural Science Foundation of China under Grant no. 62001384, the Xi'an Key Laboratory of Intelligent Perception and Cultural Inheritance under Grant no. 2019219614SYS011CG033, and also supported by the Natural Science Basic Research Plan in Shaanxi Province of China under Grant no. 2020JQ-605. It is also funded by the China Postdoctoral Science Foundation under Grant no. 2020M683694XB.

\section{References}

[1] K. J. Vinoy and R. M. Jha, Radar Absorbing Materials from Theory to Design and Characterization, Kluwer Academic Publishers, Boston, 1996. 
[2] B. A. Munk, Frequency Selective Surfaces: Theory and Design, Wiley, New York, 2000.

[3] G. H. H. Sung, K. W. Sowerby, M. J. Neve, and A. G. Williamson, "A frequency-selective wall for interference reduction in wireless indoor environments," IEEE Antennas and Propagation Magazine, vol. 48, no. 5, pp. 2937, 2006.

[4] J. Shaker, R. Chaharmir, and H. Legay, "Investigation of FSSbacked reflectarray using different classes of cell elements," IEEE Trans. Antennas Propag, vol. 56, no. 12, pp. 3700-3706, 2008.

[5] G. I. Kiani, L. G. Olsson, A. Karlsson, K. P. Esselle, and M. Nilsson, "Crossdipole bandpass frequency selective surface for energy-saving glass used in buildings," IEEE Trans. Antennas Propag, vol. 59, no. 2, pp. 520-525, 2011.

[6] R. R. Xu, H.-c. Zhao, Z.-y. Zong, and W. Wu, "Dual-band capacitive loaded frequency selective surfaces with close band spacing," IEEE Microwave and Wireless Components Letters, vol. 18, no. 12, pp. 782-784, 2008.

[7] R. Sivasamy and M. Kanagasabai, "A novel dual-band angular independent FSS with closely spaced frequency response," IEEE Microwave and Wireless Components Letters, vol. 25, no. 5, pp. 298-300, 2015.

[8] C. Sihong, P. Taisong, and L. Yuan, "Flexible dual-band ultrathin FSS with ultra-close band spacing," in 2018 International Symposium on Antennas and Propagation, pp. 1-2, IEEE, 2018.

[9] X. Sheng, J. Ge, K. Han, and X. Zhu, "Transmissive/reflective frequency selective surface for satellite applications," IEEE Antennas Wirel Propag Lett, vol. 17, no. 7, pp. 1136-1140, 2018.

[10] W. Li, C. Wang, Y. Zhang, and Y. Li, “A miniaturized frequency selective surface based on square loop aperture element," International Journal of Antennas and Propagation, vol. 2014, 6 pages, 2014.

[11] S. N. Azemi, K. Ghorbani, and W. S. T. Rowe, "Angularly stable frequency selective surface with miniaturized unit cell," IEEE Microw. Wireless Compon. Lett., vol. 25, no. 7, pp. 454456, 2015.

[12] S. Ghosh and K. V. Srivastava, "An angularly stable dual-band FSS with closely spaced resonances using miniaturized unit cell," IEEE Microwave and Wireless Components Letters, vol. 27, no. 3, pp. 218-220, 2017.

[13] H. Tao, W. Mengdan, P. Ke et al., "Ultrathin and miniaturized frequency selective surface with closely located dual resonance," IEEE Antennas and Wireless Propagation Letters, vol. 18, no. 6, pp. 1288-1292, 2019.

[14] T. Cheng, Z. Jia, T. Hong, W. Jiang, and S. Gong, "Dual-band frequency selective surface with compact dimension and low frequency ratio," IEEE Access, vol. 8, pp. 185399-185404, 2020.

[15] R. J. Langley and E. A. Parker, "Double-square frequencyselective surfaces and their equivalent circuit," Electronics Letters, vol. 19, no. 17, pp. 675-677, 1983. 